Gut, 1987, 28, 970-975

\title{
75Se HCAT test in the detection of bile acid malabsorption in functional diarrhoea and its correlation with small bowel transit
}

\author{
G SCIARRETTA, G FAGIOLI, A FURNO, G VICINI, L CECCHETTI, \\ B GRIGOLO, A VERRI, AND P MALAGUTI
}

From the Gastroenterology Unit; Department of Nuclear Medicine; Department of Health and Medical Physics; Ospedale Maggiore USL, 27; 3rd Medical Clinic, University, Bologna, Italy

SUMMARY The purpose of this study was to evaluate whether bile acid malabsorption assessed by the 75SeHCAT test, had a pathogenetic role in functional chronic diarrhoea and to ascertain whether the small bowel transit time (SBTT) could be correlated with the 75SeHCAT test results. The test was based on the counting of the abdominal retention of a 75-selenium labelled homotaurocholic acid. The 75SeHCAT test was carried out in a control group of 23 healthy adults and in 46 patients, 38 of whom were suffering from irritable bowel syndrome (IBS) of diarrhoeic form and eight patients who had undergone cholecystectomy and were suffering from chronic diarrhoea. Faecal bile acid loss was determined in nine patients, and in 14, serum bile acid increase after a standard meal was measured. In 17, SBTT was studied by hydrogen breath test after lactulose administration ( $21 \mathrm{~g}$ in $300 \mathrm{ml}$ water). In 15 patients, choledochocaecal transit time was estimated by Tc99m-HIDA (111 MBq) cholescintigraphy. In 20 of 46 subjects, 75SeHCAT retention was below normal level, and in 19 cholestyramine administration relieved diarrhoea. 75SeHCAT results were related to faecal bile acid loss, while no correlation was found with serum bile acids and SBTT. The data suggest a possible wider use of the 75SeHCAT test in chronic diarrhoea to estimate bile acid malabsorption in irritable bowel syndrome, diarrhoeic form, and provide an effective treatment. In our patients small bowel transit velocity does not seem to be a pathogenetic factor of bile acid malabsorption.

Irritable bowel syndrome (IBS) is quite a common disease in gastroenterology, and diagnosis is based on the exclusion of intestinal or extraintestinal organic pathology. Bile acids may play a pathogenetic role in chronic diarrhoea, but in practice bile acid malabsorption is not easily proved and for this very reason some consider that bile acid diarrhoea is underdiagnosed.'

Idiopathic bile acid malabsorption ${ }^{2}$ is now being investigated with renewed interest since a new radioisotopic test (75SeHCAT test) was brought into clinical practice. ${ }^{3+}$ The 75 SeHCAT test proved to be useful in the investigation of ileal pathology, ${ }^{5-7}$ and is highly sensitive and specific in bile acid malabsorp-

Address for correspondence: Dr Giuseppe Sciarretta, Servizio di Gastroenterologia, Ospedale Maggiore USL 27, 40133 Bologna, Italy.

Received for publication 8 January 1987. tion assessment. ${ }^{*}$ Clinical research has recently drawn attention to the finding of abnormally low 75SeHCAT retention in diarrhoeic patients considered to suffer from irritable bowel syndrome. ${ }^{184}$.

In this study, 75SeHCAT test was carried out in patients suffering from chronic or recurrent diarrhoea which was thought to be functional and to investigate whether bile acid malabsorption had a pathogenetic role in this syndrome. The results of the 75SeHCAT test were correlated with SBTT and other parameters of bile acid absorption.

\section{Methods}

PATIENTS

The study was carried out under the Helsinki

Declaration. Forty six patients were studied, all of 
whom had given written informed consent. Twenty were male, 26 female, aged between 17 and 73 years (mean 41). In 38 cases diagnosis of irritable bowel syndrome was established, according to GudmandHoyer et al," based on the clinical picture and the absence of organic diseases affecting the alimentary tract. In the other eight, a cholecystectomy had already been done. Chemical and microbiological faecal analysis showed normal results. Radiographic examinations of large and small bowel, carried out using two contrast media were negative. Diabetes, other endocrine diseases, and food allergies were excluded, and all the patients were on a lactose free diet. The clinical picture was characterised by chronic or recurrent diarrhoea with liquid or semiliquid stools. The general nutritional condition appeared good, however, abdominal pain was often present and anticholinergic agents, loperamide and high fibre diet had not relieved the symptoms. In six of eight cholecystectomised patients diarrhoea occurred after surgery, while in the other two it was already present.

Different methods were used to investigate bile acid malabsorption and small bowel transit.

\section{SeHCAT TEST}

The 75SeHCAT test was carried out in all patients using the method we described elsewhere and the control group consisted of the same 23 subjects. ${ }^{*}$ Results are expressed as percentage retention values calculated by the exponential time activity curve on day 3. Measurements of abdominal radioactivity were taken by gamma camera counting on the day of administration of $370 \mathrm{KBq} 75 \mathrm{Se}$-homocholyltaurine (75SeHCAT, Amersham Radiochemical Centre, England) (time zero) and on days 1-3-5 and 7. An abdominal retention of $34 \%$ or more on day 3 is considered normal by our method. ${ }^{x}$. The percentage abdominal retention on day 7 , measured directly by gamma camera for both the control and the functional diarrhoea groups was considered. An abdominal retention of less than $8 \%$ (the lowest value in a normal subject) is considered pathologic.

\section{CHOLESTYRAMINE TEST}

Cholestyramine was given to all patients twice daily to give a total dose of 2-8g/day for a period of at least 10 days. When cholestyramine was not effective in relieving symptoms, therapy was discontinued. In those patients where the drug was effective, it was stopped for seven days, and started again if diarrhoea recurred. The test was considered positive when diarrhoea stopped with cholestyramine administration, and recurred without it. In all patients stool frequency which was taken as the average number of bowel actions per day, over a period of one week, was recorded for the week preceding and after administration.

POSTPRANDIAL SERUM BILE ACID RESPONSE In 14 patients, conjugated bile acids (cholic, chenodeoxycholic and deoxycholic acid) were tested in serum by a radioimmunologic method (Farmos Diagnostica, Finland). Accuracy of the method is between $90 \%$ and $110 \%$ and sensitivity is 0.2 $\mu \mathrm{mol} / \mathrm{l} . "{ }^{12}$ Blood samples were taken at $-15 \mathrm{~min}$, just before the ingestion of a standard meal $(900 \mathrm{cal})$, and $60,90,120,150,180 \mathrm{~min}$ after eating. Meal ingestion started at midday, according to Aldini et $a l^{1.3}$ for a better sensitivity of the test. The maximum increase $(\triangle)$ after meal of each bile acid concentration was used for correlation with the $75 \mathrm{SeHCAT}$ test results.

FAECAL BILE ACID DETERMINATION

Faecal bile acid loss was measured in nine patients after three day stool collection. For measuring faecal bile acid, $1 \mathrm{~g}$ faeces taken from each sample was dissolved in $10 \mathrm{ml}$ iso-propyl alcohol. ${ }^{14}$ After centrifugation at $2500 \mathrm{~g}$ for $15 \mathrm{~min}, 2-5 \mathrm{ml}$ supernatant was put into test tubes and dried under nitrogen stream. The dry residue was mixed with $5 \mathrm{ml} \mathrm{NaOH} 0 \cdot 1 \mathrm{M}$ and the solution was passed through Bond-Elut C18 columns which were consecutively activated with 3 $\mathrm{ml}$ methanol and $6 \mathrm{ml} \mathrm{H}_{2} \mathrm{O}$ and finally eluted with $4 \mathrm{ml}$ methanol. The sample was again dried under nitrogen stream, after which it was ready for bile acid assay. Total bile acid determination was carried out by an enzymatic fluorimetric method ( $3 \alpha \mathrm{HSD})$. A faecal bile acid amount less than $250 \mathrm{mg} /$ day was considered normal. ${ }^{1+15}$

SMALL BOWEL TRANSIT TIME

Small bowel transit time (SBTT) was assessed in 17 patients by hydrogen breath test $\left(\mathrm{H}_{2}-\mathrm{BT}\right)$ according to the technique of Metz et al ${ }^{16}$ after oral administration of an isotonic solution of lactulose $(21 \mathrm{~g}$ lactulose in $300 \mathrm{ml}$ water). Alveolar air samples for hydrogen content measurement were collected at five minute intervals for two hours or until a marked increase of $\mathrm{H}_{2}$ concentration was observed. An increase of $\mathrm{H}_{2}$ concentration of at least $20 \mathrm{ppm}$ above the basal value means that lactulose has reached the caecum and defines SBTT. ${ }^{17}$ Two patients were $\mathrm{H}_{2}$-nonproducers, therefore the SBTT could only be ascertained in 15 patients.

RADIOISOTOPIC DETERMINATION OF CHOLEDOCHOCAECAL TRANSIT TIME

Because 75SeHCAT travels in the bowel mixed with bile, choledochocaecal transit time was measured by Tc99m-HIDA cholescintigraphy in 15 patients. For 
this purpose the patient received $111 \mathrm{MBq} \mathrm{Tc} 99 \mathrm{~m}$ HIDA intravenously 15 minutes after oral ingestion of a fat meal $(200 \mathrm{ml}$ cholecystokinetic standard meal) to empty the gall bladder. In this way, bile flows into the duodenum through the choledochus, with minor influence from gall bladder filling and moreover cholecystectomised subjects can be studied using the same method. Images of the abdominal radioactivity were collected by a large field of view gamma camera and fed into a computer, at a rate of 1 frame per minute in $128 \times 128$ bite matrix. Data acquisition started at the moment of the injection and lasted until the radiocompound reached the caecum and the ascending colon. On the computer stored images, regions of interest which were the choledochus, the caecum and the ascending colon were outlined, and the corresponding time activity histograms generated. ${ }^{18}$ Choledochocaecal transit time was the time that elapsed between the visualisation of the choledochus and the appearance of the radioactivity in the caecum.

\section{Results}

The 75SeHCAT retention values shown on the curve on day 3 and from the gamma camera counting on day 7 are shown in Figure 1. From the curve, 20

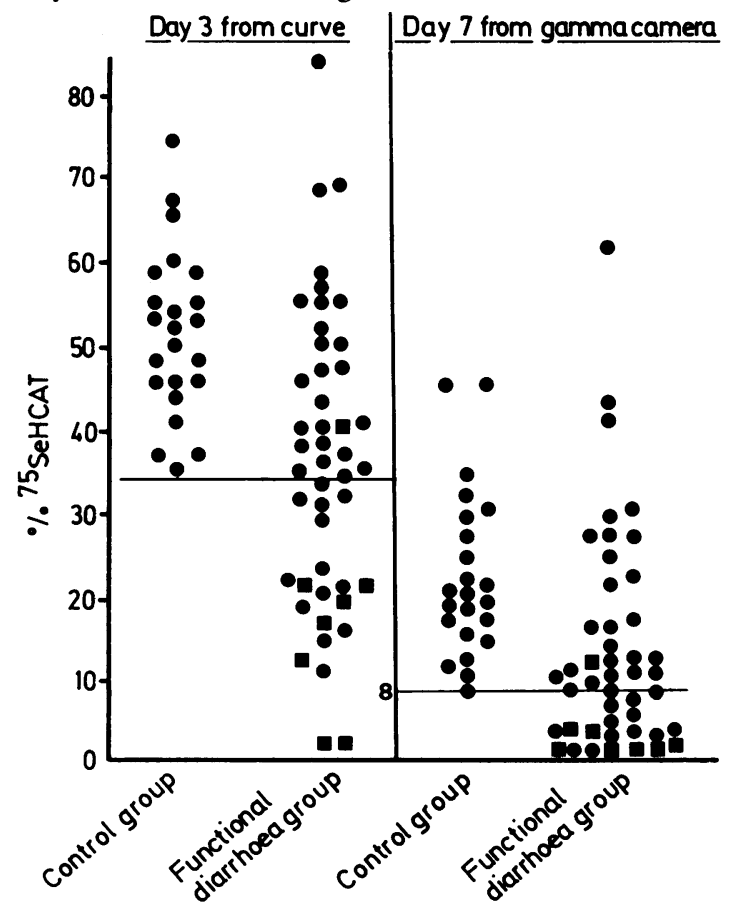

Fig. 1 75SeHCAT values of the 46 patients studied and of the 23 normal controls. Horizontal lines represent, in all figures, the lower normal limit of $75 \mathrm{SeHCAT}$ retention. Squares mark cholecystectomised patients, in all figures. patients transpired to be within abnormal range on day 3 , while from the gamma camera 18 appeared on day 7 and the other two cases had a retention of $8 \%$ which is the lower limit of normal level. Only one of 8 cholecystectomised patients (marked by squares) had a normal 75SeHCAT retention. The effects of cholestyramine administration on stool frequency, both in normal and in low 75SeHCAT retention patients, are shown in Figure 2 . In 19 of 20 patients with low 75SeHCAT, cholestyramine was effective in relieving diarrhoea, while 25 of 26 cases with normal 75 SeHCAT retention had no positive response. In Figure 3 the results of the 75SeHCAT test and the maximum increase of serum cholic acid, which is almost completely reabsorbed by the distal ileum, are correlated. No correlation was found between the two tests nor between the 75SeHCAT values and the other two serum bile acids. Total faecal bile acids results, with the corresponding 75SeHCAT values, are shown in Figure 4. The four cases with normal 75SeHCAT retention had normal faecal bile acid loss, while the five patients with low 75SeHCAT retention had increased faecal bile acid loss. In Figure 5 SBTT, measured by $\mathrm{H}_{2}-\mathrm{BT}$ and the corresponding values of the 75SeHCAT test are shown. There is no correlation between the two examinations. Figure 6 represents choledochocaecal transit time measured by cholescintigraphy and the corresponding results of 75SeHCAT test and no correlation was observed.

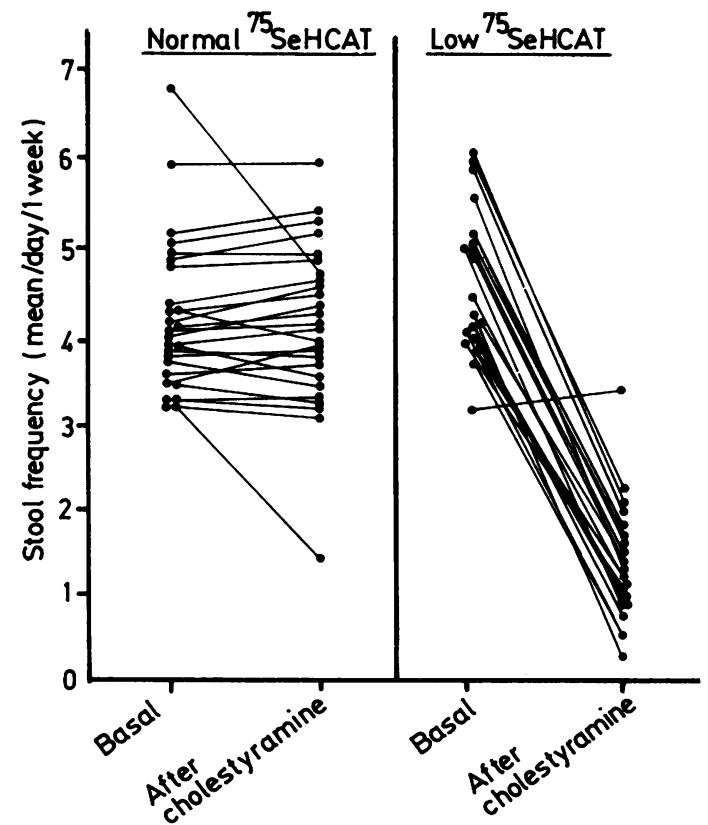

Fig. 2 Stool frequency changes after cholestyramine in patient with normal and low $75 \mathrm{SeHCAT}$ retention. 


\section{Discussion}

The 75SeHCAT test, recently introduced in clinical practice, has made the detection of bile acid malabsorption more easily detectable. In Crohn's ileitis and ileal resections, the 75SeHCAT test showed a high diagnostic accuracy both when the test was done

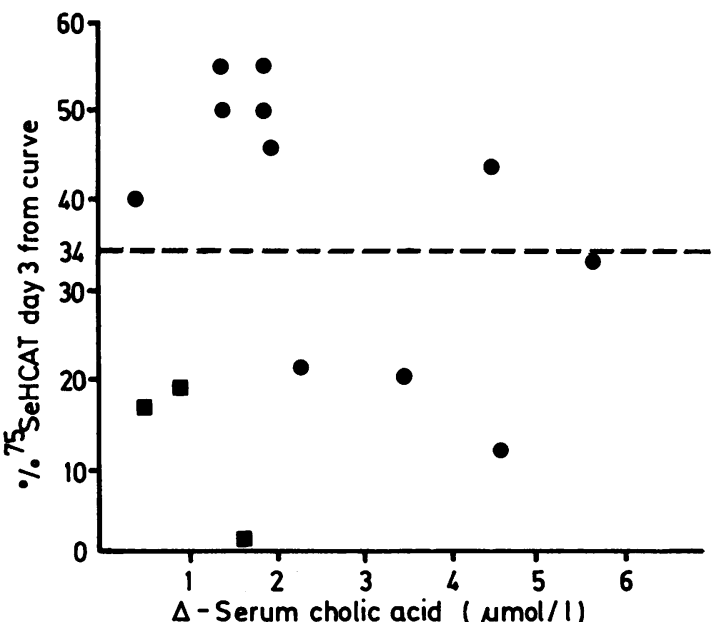

Fig. 3 Maximum postprandial increase of conjugated cholic acid and 75SeHCAT values: no correlation is present.

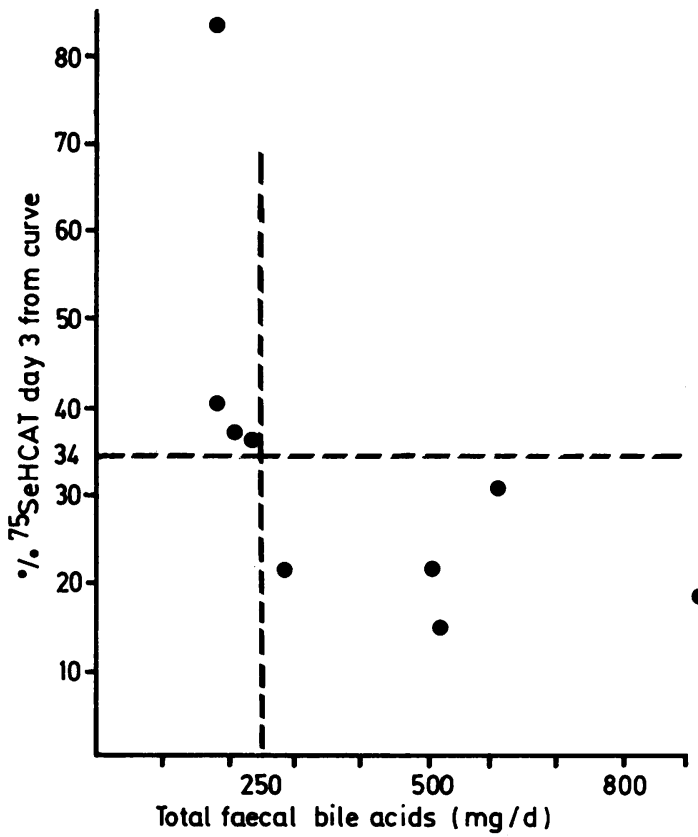

Fig. 4 75SeHCAT values and total faecal bile acids of nine patients. Vertical dotted line marks the lower normal limit for total faecal bile acids ( $250 \mathrm{mg} /$ day). using a whole body counter ${ }^{+}$and when abdominal retention was measured by gamma camera. ${ }^{5 x}$ As the test is easy to do and causes the patient no discomfort, it can be widely used to investigate irritable bowel syndrome, diarrhoeic form, which is a common pathological condition. Diagnosis of irritable bowel syndrome relies on the exclusion of any demonstrable disease, but bile acid malabsorption which was at one time difficult to detect, seems to play an important pathogenetic role in chronic and recurrent diarrhoea. Some investigators are of the opinion that bile acid diarrhoea is currently underdiagnosed, and many cases where diarrhoea is caused by bile acid malabsorption are erroneously attributed to the diarrheic form of irritable bowel syndrome. ${ }^{1 \times 9}$

In patients affected by chronic or recurrent diarrhoea we carried out the 75SeHCAT test which showed evidence of an increased bile acid loss in more than $40 \%$ of our cases. In those cases where total faecal bile acids were tested, the 75SeHCAT results were also consistent, thus confirming that an abnormally low retention value of $75 \mathrm{SeHCAT}$ corresponds to an increased bile acid loss. In 19 of 20 patients with a pathologic $75 \mathrm{SeHCAT}$ test, a positive clinical response to cholestyramine confirmed that bile acid malabsorption played an important role in causing diarrhoea. In one patient whose 75SeHCAT value was $32 \%$, cholestyramine was not able to

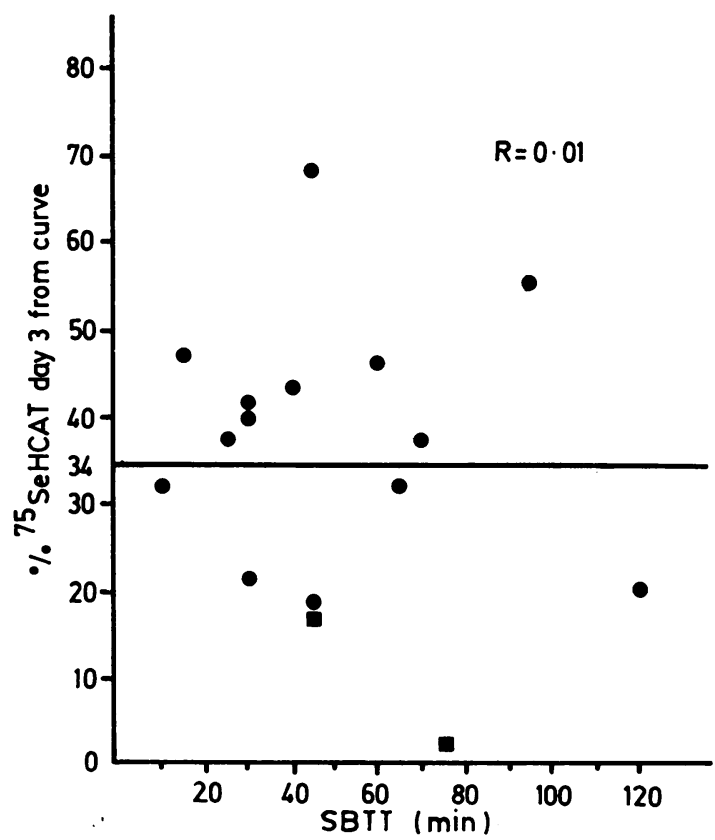

Fig. 5 Small bowel transit time, measured by hydrogen breath test with lactulose, and 75SeHCAT values of 15 patients: no correlation found. 


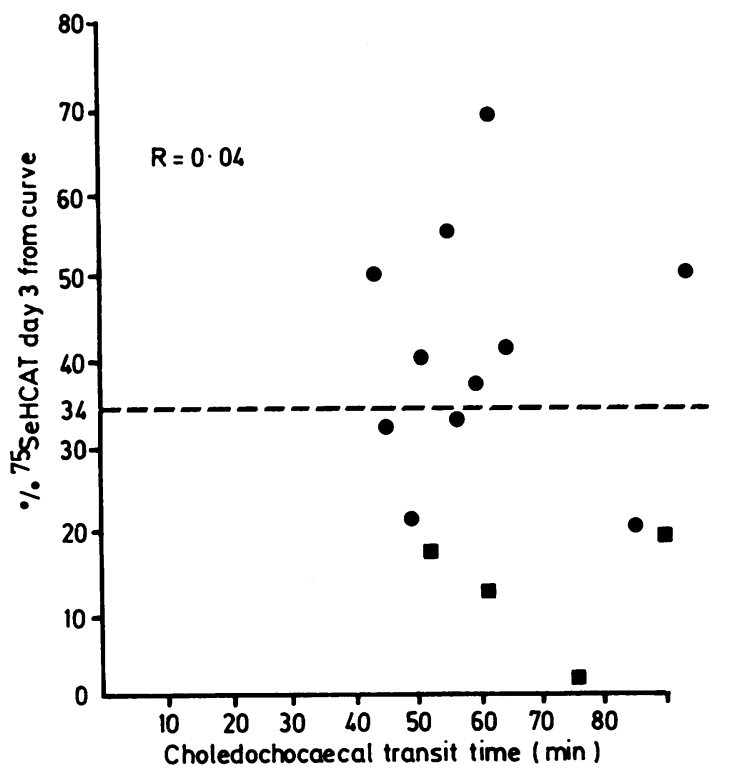

Fig. 6 Choledochocaecal transit time, studied by cholescintigraphy with Tc $99 \mathrm{~m}-\mathrm{HIDA}$, and 75SeHCAT results in 15 patients: no correlation observed.

control the symptoms. The low doses of cholestyramine sufficient enough to control diarrhoea (2-8 $\mathrm{g} /$ day) are justified by the moderate bile acid malabsorption observed in these patients who were diarrhoeic patients, but not to the same extent as some Crohn's ileitis or ileum resected patients where the 75SeHCAT retention is very low and often zero on the third day. ${ }^{8}$ Determination of serum bile acids is not a sensitive test when undertaken to detect bile acid malabsorption in patients with chronic or recurrent functional diarrhoea, where bile acid malabsorption is not severe. Moreover bile acid concentrations in serum do not depend on intestinal absorption alone, but many other factors are involved including principally liver function. Diarrhoea caused by idiopathic bile acid malabsorption is a well known physiopathological entity, but it is currently considered to be uncommon. We estimate moderate bile acid malabsorption to be much more frequent in patients suffering from functional diarrhoea. More than $40 \%$ of our patients had a pathologic low 75SeHCAT retention. Our results are consistent with the opinion of Merrick et al' that bile acid malabsorption as a cause of diarrhoea is underestimated. In the population they examined, however, bile acid malabsorption was present in a lower percentage of patients: $12 \%$. To explain this difference three factors may be taken into account: (1) none of the patients studied by Merrick et al was cholecystectomised: eight of our patients were, and in seven the 75SeHCAT test was abnormal; in these subjects, an increased frequency of enterohepatic cycling and a greater postprandial bile load into the ileum may have been involved. (2) In the valuation of the 75SeHCAT test, Merrick et al considered a 'range of values of uncertain attribution ...', but a different approach was used here, and results were considered to be either normal or abnormal. (3) Nutritional and racial differences may also play a role.

As far as the mechanism of bile acid malabsorption is concerned, three situations may be considered in patients with intact gall bladder function: (1) the efficiency of distal ileum mucosa in reabsorbing bile salts is reduced; (2) an accelerated transit of intestinal content in the distal ileum reduces the time for bile salts reabsorption; (3) the enterohepatic cycling frequency may be increased as in cholecystectomised subjects. In this study, small bowel transit time was investigated by means of hydrogen breath test after lactulose ingestion and cholescintigraphy. Lactulose solution, used to measure small bowel transit time ${ }^{17}$ ix may not be considered a physiological meal and it passes through the small bowel more quickly than a solid meal. Thus a difference between those patients with and without bile acid malabsorption may be concealed, and further studies should be carried out using more physiological meals. In patients with a pathologic 75SeHCAT test, in which bile acid malabsorption is present, these two methods did not show a decreased SBTT or choledochocaecal transit time, and no correlation was found between these two results and the 75SeHCAT test result. No correlation was found, either between SBTT and choledochocaecal transit time which were both done in 11 patients. This may be because of both the wide variability of the results and of the different mechanisms of the two tests.

On the basis of these data, we conclude that $75 \mathrm{SeHCAT}$ test, systematically carried out in patients suffering from chronic or recurrent diarrhoea, is able to detect those cases where bile acid malabsorption is present and a diagnosis of irritable bowel syndrome would not be correct. Moreover, although the processes involved are unknown, bile acid malabsorption seems to be unrelated to small bowel transit velocity in our patients.

\section{References}

1 Merrick MV, Eastwood MA, Ford MJ. Is bile acid malabsorption underdiagnosed? An evaluation of accuracy of diagnosis by measurement of SeHCAT retention. Br Med J 1985; 290: 665-8.

2 Thaysen EH, Pedersen L. Idiopathic bile acid catharsis. Gut 1976; 17: 965-70.

3 Merrick MV, Eastwood MA, Anderson JR, Ross HM. Enterohepatic circulation in man of gamma-emitting 
bile acid conjugate, 23-selena-25-homotaurocholic acid (SeHCAT). J Nucl Med 1982; 23: 126-30.

4 Nyhlin H, Merrick MV, Eastwood MA, Brydon WG. Evaluation of ileal function using 23-selena-25homotaurocholate, a $\delta$-labelled conjugated bile acid. Gastroenterology 1983; 84: 63-8.

5 Thaysen EH, Orholm M, Arnfred T, Carl J, Rodbro P. Assessment of ileal function by abdominal counting of the retention of a gamma emitting bile acid analogue. Gut 1982; 23: 862-5.

6 Fagan EA, Chadwick VS, Baird IMcL. SeHCAT absorption: a simple test of ileal dysfunction. Digestion 1983; 26: 159-65.

7 Holdstock G, Phillips G, Hames TK, et al. Potential of SeHCAT retention as an indicator of terminal ileal involvement in inflammatory bowel disease. Eur J Nucl Med 1985; 10: 528-30.

8 Sciarretta G, Vicini G, Fagioli G, Verri A, Ginevra A, Malaguti P. Use of 23-selena-25-homocholyltaurine to detect bile acid malabsorption in patients with ileal dysfunction or diarrtea. Gastroenterology 1986; 91: 1-9.

9 Heaton KW. Functional diarrhoea: the acid test. $\mathrm{Br}$ Med J 1985; 290: 1298-9.

10 Gudmand-Hoyer E, Riis P, Wulff HR. The significance of lactose malabsorption in the irritable colon syndrome. Scand J Gastroenterol 1973; 8: 273-8.

11 Maentausta O, Janne O. Radioimmunoassay of con- jugated cholic acid, chenodeoxycholic acid, and deoxycholic acid from human serum, with use of $125 \mathrm{I}$ labelled ligands. Clin Chem 1979; 25: 264-68.

12 Kallberg M, Tobiasson P. Determination of cholic and chenodeoxycholic acid in serum: evaluation of two commercial radioimmunoassay methods. J Clin Chem Clin Biochem 1980; 18: 491-95.

13 Aldini R, Roda A, Festi D, et al. Diagnostic value of serum primary bile acids in detecting bile acid malabsorption. Gut 1982; 23: 829-34.

14 Colombo C, Roda A, Valade A, Piceni Sereni L. Faecal bile acid extraction in childhood. Riv Ital Pediatr 1982;8: 77-85.

15 Aldini R, Roda A, Festi D, et al. Bile acid malabsorption and bile acid diarrhoea in intestinal resection. Dig Dis Sci 1982; 27: 495-502.

16 Metz G, Gassull MA, Leeds AR, Blendis LM, Jenkins DJA. A simple method of measuring breath hydrogen in carbohydrate malabsorption by end-expiratory sampling. Clin Sci Mol Med 1976; 50: 237-40.

17 Bond JH, Levitt MD, Prentiss R. Investigation of small bowel transit time in man utilizing pulmonary hydrogen $\left(\mathrm{H}_{2}\right)$ measurements. J Lab Clin Med 1975; 85: 546-55.

18 Caride VJ, Prokop EK, Troncale FJ, Buddoura W, Winchenbach K, McCallum RW. Scintigraphic determination of small intestinal transit time: comparison with the hydrogen breath technique. Gastroenterology 1984; 86: 714-20. 